\title{
Lysosomal Acid Lipase Activity Deficiency in Children with Liver Disease: a potential biomarker?
}

\author{
Giusy Ranucci*1,2, Giada Zollo ${ }^{1}$, Giulia Tozzi $^{3}$, Valerio Nobili ${ }^{4,5}$, Maria Immacolata Spagnuolo ${ }^{1}$ and \\ Raffaele Iorio ${ }^{1}$
}

${ }^{1}$ Department of Translational Medical Science, Section of Pediatric, University of Naples Federico II, Italy

${ }^{2}$ Division of Metabolism, Bambino Gesù Research Children's Hospital, Rome, Italy

${ }^{3}$ Laboratory of Molecular Medicine, Unit of Neuromuscular and Neurodegenerative Disorders, Bambino Gesù Children's Hospital, IRCCS, Rome, Italy

${ }^{4}$ Hepatology, Gastroenterology and Nutrition, Bambino Gesù Children's Hospital, IRCCS, Rome, Italy

${ }^{5}$ Department of Pediatric, University la Sapienza Rome

Received: June 13, 2018; Published: July 02, 2018

*Corresponding author: Giusy Ranucci, Bambino Gesù Research Children Hospital, Division of Metabolism, Piazza di Sant’0nofrio, 4, 00165 Rome, Italy

\section{Abstract}

Background: While a total Lysosomal Acid Lipase activity deficiency determines Wolman Disease, in partial deficiency there's a residual enzyme activity. Onset and clinical features of partial LAL-d are very variable. Secondary LAL-d has been found in patients with non alcoholic fatty liver disease obesity related. The aim of this study was to evaluate Lysosomal Acid Lipase activity (LAL-A) deficiency determined by dried blood spotting (DBS) in pediatric patients with known and unknown liver disease in order to clarify the role of this test in pediatric clinical practice. Relationship between LAL-A and liver enzymes, dyslipidemia and other parameters of metabolic syndrome was investigated.

Results: We evaluated 51 patients ( 36 males) with a median age of 13 years, 25 subjects with a liver disease of unknown origin and 26 with a known liver disease. LAL-A was determined by DBS on filter paper; specific genetic analysis was performed in patients with LAL-A less then the lower limit of normal. No patient resulted affected by genetically determined LAL deficiency. A low LAL-A at DBS was found in 11 (22\%) patients (8 males) with variable reduction of enzymatic activity from $15 \%$ to $50 \%$ of the lower limit of normal. On ultrasound, liver steatosis was found in all patients with LAL deficiency. A significant inverse correlation between LAL-A and age of patients, triglycerides and uric acid serum levels was found. Low LAL-A subjects had significantly higher triglycerides and uric acid levels than normal one. Finally, Wilson Disease (WD) patients with low LAL-A had body-mass-index, total cholesterol, triglycerides and uric acid levels higher than normal LAL-A WD patients.

Conclusion: The study shows that different liver diseases may affect LAL activity that is inversely related to the age of patients and both triglycerides and uric acid serum levels. LAL-A is reduced in a subgroup of WD patients that showed higher BMI, total cholesterol, triglycerides and uric acid levels.

Keywords: Cholesterol Esteryl Storage Disease; Steatosis; Dyslipidaemia; Wilson Disease; Metabolic Syndrome

Abbreviations: LAL: Lysosomal Acid Lipase; LIPA: Lipase A; LAL-A: LAL Activity; DBS: Dried Blood spots; NAFLD: Non Alcoholic Fatty Liver Disease; NASH: Non Alcoholic Steatohepatitis; ALT: Alanine Aminotransferase; BMI: Body Mass Index; HOMA-IR: Insuline Resistance Index; NAS: NAFLD Activity Score; WD: Wilson Disease; BA: Biliary Atresia; AIH: Auto Immune Hepatitis; INR: International Normalised Ratio; US: ultrasound; GGT: Gamma-glutamyltransferase; PT: Prothrombin Time; aPTT: Activated Thromboplastin Time; T-C: Total Cholesterol; LDL-C: LDL-cholesterol; HDL-C: HDL-cholesterol; PNPLA3: Patatin-Like Phospholipase Domain; PCR: Polymerase Chain Reaction

\section{Introduction}

The deficit of lysosomal acid lipase (LAL) is a rare autosomal recessive lysosomal storage disease, deriving from mutations in the Lipase A (LIPA) gene on chromosome 10. The total enzyme activity deficiency (activity lower than 1\% of normal range or totally absent activity) is associated with Wolman Disease which is characterized by an early onset in the first weeks of life with gastrointestinal symptoms such as vomiting, diarrhoea with steatorrhoea, anaemia and abdominal distension due to massive hepatosplenomegaly. The liver disease can evolve into cirrhosis and/or liver failure [1,2]. All of these signs and symptoms derive from lysosomal and macrophagic storage of triglycerides and cholesteryl esters in many organs like liver, spleen, adrenal glands, bone marrow, lymph nodes and intestinal villi. In patients with partial deficiency of LAL the onset is variable and the clinical features are less well defined, depending on the degree of residual activity of the enzyme.

In presence of a residual enzyme activity, clinical features are less severe and the patients usually show hepatomegaly, raised aminotransferase serum levels, high serum levels of triglycerides 
and LDL-cholesterol with low or normal levels of HDL-cholesterol (type IIb dyslipidemia). These non-specific clinical and laboratory signs make the disease very difficult to be diagnosed during childhood, so that a correct diagnosis is often made in adult age and, moreover, with important complications: indeed, lipid storage in liver can cause the development over time of hepatic fibrosis and micronodular cirrhosis [3-5]. On the other hand, in these subjects hyperlipidemia leads to accelerated atherosclerosis and therefore to an increased incidence of early cardiovascular and cerebrovascular diseases [6]. The current golden standard for diagnosis is the sequencing of coding regions of LIPA gene. Testing LAL activity (LAL-A) on Dried Blood spots (DBS), as described by Hamilton [7], seems a good strategy for a wide screening of the disease in general population.

An open question, currently debated in literature, is whether other liver diseases can affect the activity of LAL and in what extent, resulting in a secondary enzyme deficiency. Two recent studies in literature pointed out that in adult and pediatric patients with Non Alcoholic Fatty Liver Disease (NAFLD) an enzyme deficiency can be detected through DBS on filter paper. In particular, Baratta et al. showed that in adult subjects a reduced LAL-A is related to higher serum levels of transaminases and cholesterol. Moreover, LAL-A progressively decreases from patients with steatosis to those with Non Alcoholic Steatohepatitis (NASH), and an enzyme activity below the median value has a positive association with Alanine Aminotransferase (ALT) serum levels and the presence of NASH [8]. Similar evidences were reported in children with NAFLD by Selvakumar et al., who found a negative correlation between LAL-A and Body Mass Index (BMI) z-score, total cholesterol, LDLcholesterol and Insuline Resistance Index (HOMA-IR); furthermore, enzyme activity was lower in children with significant fibrosis than those with mild fibrosis [9].

These results suggest that in both adult and pediatric subjects a deficient LAL-A is strictly related to a clinical and laboratory profile of metabolic syndrome and of a more severe liver disease $[8,9]$. A correlation between LAL deficiency and severe liver injury has also been observed in patients with hepatic cirrhosis by Shteyer and other authors [10]. In this study, low LAL-A was found in association with lower levels of platelets and white cell blood count (WBC) (markers of portal hypertension) and with higher serum levels of creatinine and uric acid (markers of hepatorenal syndrome) and of glucose and glycosylated hemoglobin (markers of metabolic syndrome). LAL deficiency was also related to steatosis, hepatomegaly and higher grade of fibrosis assessed by NAFLD Activity Score (NAS). Authors speculated that low LAL-A in patients with severe liver disease is merely a consequence of an overall decrease in viable hepatocytes that leads to lower protein production [10]. On the other hand, various studies in animal models suggest that lower LAL-A may be part of the pathogenesis of fatty liver disease [10]. In the present study we evaluate the prevalence of LAL-A deficiency in pediatric patients with known and unknown liver disease in order to clarify the role of this test in pediatric clinical practice.

\section{Methods}

\section{Setting of the Study}

We evaluated 51 patients (36 males), followed at the Pediatric Liver Unit of University "Federico II" of Naples from 1986 to 2015, affected by different liver diseases, diagnosed during childhood. Studied group had a mean age of 13 years (range 2-32 years) at the time of the study.

Twenty-five patients had a liver disease of unknown origin (first group). In this group 20 patients showed high cholesterol levels and/or sonographic signs of hepatic steatosis and 5 patients had histological signs of steatohepatitis. These patients were extensively studied for common causes of liver disease without a final diagnosis. Six of these patients have a BMI greater than 85 percentiles, but a final diagnosis of NAFLD was not supported because of persistence of hypertransaminasemia despite weight loss. On the basis of these criteria, these patients were considered eligible for DBS screening to evaluate LAL-A.

Twenty-six patients had a definite liver disease (second group):
a) Wilson Disease (WD) in 20
b) Biliary Atresia (BA) with a fibrosis stage 3-4 at liver biopsy in 4

c) Autoimmune hepatitis (AIH) with a fibrosis stage 3-4 at liver biopsy in 2 .

\section{Study Design}

Data were collected from June 2014 to June 2015. Study protocol was approved by Ethical Committee board of University Federico II and parental consent was obtained. Inclusion criteria were: liver disease, absence of liver failure as documented by normal values of albumin and of International Normalised Ratio (INR), absence of LAL data before study enrollment, laboratory markers of liver disease and metabolic syndrome availability, ultrasound parameters availability.

The study design included:

a) Evaluation of health conditions through anamnesis and physical examination

b) Recording of anthropometric parameters (weight, height and BMI) using 2000 CDC growth charts by Kuczmarski et al. [11]

c) Recording of longitudinal diameter of liver and spleen and detection of hepatic steatosis at abdomen ultrasound (US) evaluation (US images of patients were retrieved from the institutional PACS). A board-certified radiologist, who was blinded to the pathology results and clinical history, reviewed the US examinations. The cases were subjectively classified as no steatosis, mild steatosis (increased echogenicity of the liver), moderate steatosis (some loss of visibility of internal vascular architecture and mild sound attenuation in the presence of 
echogenic liver), or severe steatosis (marked sound attenuation leading to poor visualization of the diaphragm and intrahepatic vessels).

d) Recording of laboratory markers of liver disease (Aspartate Aminotransferase (AST), ALT, Gamma-glutamyltransferase (GGT), bilirubin, albumin, prothrombin time (PT), activated thromboplastin time (aPTT)) and metabolic syndrome (glucose, triglycerides, total cholesterol (T-C), LDL-cholesterol (LDL-C), HDL-cholesterol (HDL-C), insulin, glycosylated hemoglobin) on serum samples collected with standard methods.

e) Determination of LAL-A on dried blood spots on filter paper according to the Hamilton's method [7].

f) Determination of genetic analysis for patients with LAL activity deficiency.

\section{DBS Preparation and LAL Activity Assessment}

Patients' blood spots were collected with a standard procedure: first a peripheral venous blood sample was taken, and then $75 \mathrm{ul}$ of blood was collected falling on dedicated area of filter paper. To obtain dried blood spots, papers were kept first at ambient temperature for 24 hours. Samples were stored double-bagged with desiccant at $-20{ }^{\circ} \mathrm{C}$ and analyzed within 1 week of storage. LAL-A is measured using the fluorimetric substrate 4-methylumbelliferyl palmitate in the presence of cardiolipin as an activator of LAL. on DBS was determined by using 4-methylumbelliferyl palmitate as substrate [7]. As other lipases may interfere with LAL-A measurement, a specific LAL inhibitor known as Lalistat 2 was used. Total lipase activity was determined in absence and then in presence of the inhibitor: the difference between these results corresponds with LAL-A. The range of LAL-A was considered normal between 0,73 and 3, $03 \mathrm{nmol} / \mathrm{spot} / \mathrm{h}[7,8]$. LAL activity in homozygotes patients with LAL-deficiency was $<0.03 \mathrm{nmol} / \mathrm{spot} / \mathrm{h}$ and in heterozygotes in the range $0.15-0.40 \mathrm{nmol} / \mathrm{spot} / \mathrm{h} .7 \mathrm{In}$ cases of LAL-A less than the lower limit of normal of 0.73 nomol/spot/h, a second confirmation was obtained before proceeding to genetic analysis. DBS tests were performed in Bambino Gesù Hospital in Rome. Physicians analyzing LAL activity were unaware of clinical and biochemical characteristics of any enrolled patient.

\section{Molecular Analysis}

EDTA blood of patients with a LAL activity less than lower limit of normal was collected. Then each patient's DNA was extracted from WBC using standard procedures and subjected to an individual genotyping test. Molecular analysis was made by searching mutations in LIPA gene, using polymerase chain reaction (PCR) procedure.

\section{Statistical Analysis}

Continuous variables were presented using numbers of patients $(\mathrm{N})$, means, medians and standard deviations (SD), while discrete variables were presented using percentages (\%).
Continuous variables normally distributed were presented as mean and SD and were compared between groups by ANOVA test with post-hoc testing using Scheffe's tests. Continuous variables not normally distributed were presented as median and range and were compared between groups with Kruskal-Wallis ANOVA with post-hoc testing using Mann-Whitney U-test. The linear regression was performed using Graph Pad Prism version 7.00 for Mac. A statistically significant difference was assessed for $\mathrm{p}<0,05$. For the comparative analysis, cohort was divided in patients with LAL activity less than the lower limit of normal (low LAL-A) and in those with normal enzyme activity (normal LAL-A).

\section{Aims}

Primary aims of our study were:

a) To identify the prevalence of genetically determined LAL deficiency in children with liver disease of unknown origin;

b) To evaluate the activity of LAL by DBS in patients with known liver disease such as WD, BA and AIH;

c) To evaluate the existence of a relationship between LAL activity by DBS, ALT levels and parameters of metabolic syndrome.

Secondary aim was to compare serum levels of ALT, triglycerides, T-C, LDL-C, HDL-C, uric acid, insulin, BMI and HOMA index:

a) In subjects with chronic liver disease with low levels of LAL (low LAL-A) and in those with normal enzyme activity (normal LAL-A);

b) In WD patients with low levels of LAL on DBS (low LAL-A) and in WD patients with normal enzyme activity (normal LAL-A).

\section{Results}

Median blood LAL-A in 51 enrolled patients was 0.83 (range, 0.22- 2.8). No difference between males (n: 35) and females (n: 16) $[0.97(0.62-1.75)$ vs. $0.89(0.34-2.8) p=0.63]$ was found. A low LAL -A at DBS was found in 11 (22\%) patients (8 males; median age 16 years, range 8-32 years) with variable reduction of enzymatic activity from $15 \%$ to $50 \%$ of the lower limit of normal: 3 patients (median age 14 years, range 11-16 years) belonged to the first group; 8 patients (median age 16 years, range 8-18 years) belonged to the second group ( 6 patients with WD and 2 with BA). None of these patients resulted affected by genetically determined LAL deficiency. LAL-A by DBS was inversely related to the age of the patients with liver disease $\left(r=0.34 ; r^{2}: 0.11 ; P: 0.01\right)$ (Figure 1). Furthermore LAL-A was inversely related with both triglycerides ( $\mathrm{r}$ : 0.29; $r^{2}$ : 0.08; P: 0.03) (Figure 2) and uric acid serum levels (r: 0.33; $r^{2}: 0.11 ; P: 0.01$ ) (Figure 3). No correlation between LAL activity by DBS and BMI, ALT, glucose, T-C, LDL-C, HDL-C, insulin and HOMA index was found. Ultrasound signs of steatosis were found in all patients with low LAL-A by DBS (Figure 3) Table 1. Summarized mean LAL-A and triglycerides levels according to steatosis. 


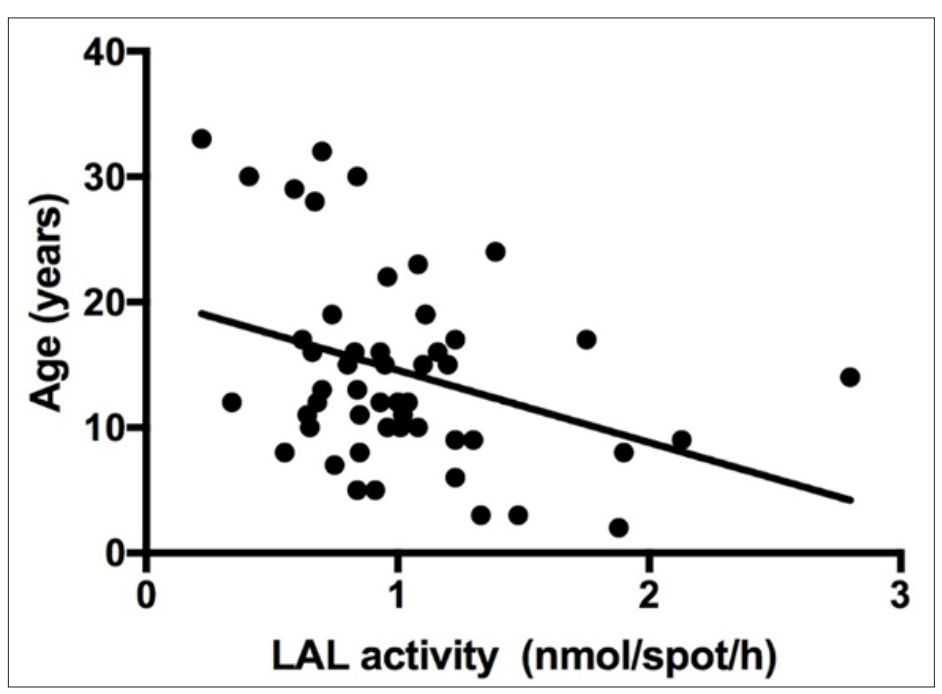

Figure 1.

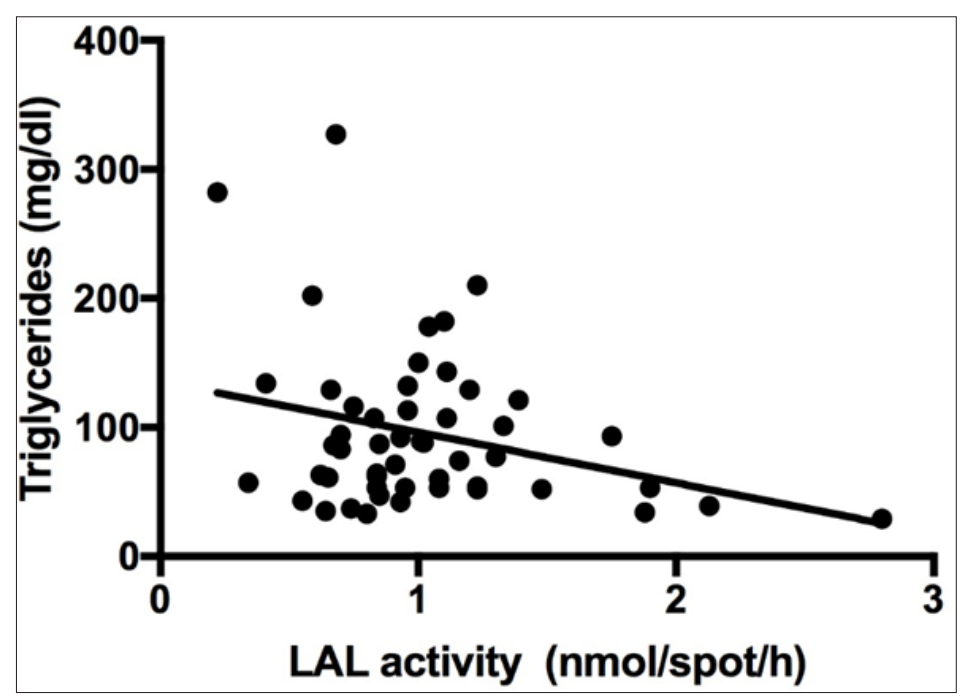

Figure 2.

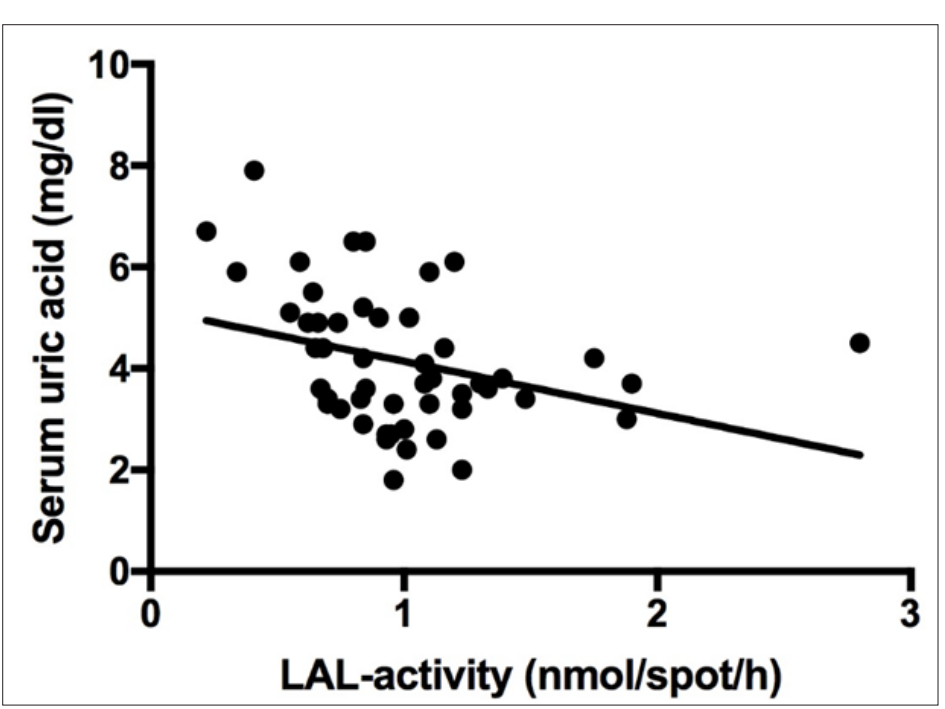

Figure 3. 
Table 1: LAL activity and Triglycerides according to steatosis.

\begin{tabular}{|c|c|c|c|c|}
\hline Parameter & $\begin{array}{c}\text { No } \\
\text { steatosis } \\
\text { (n: 25) }\end{array}$ & $\begin{array}{c}\text { Mild } \\
\text { steatosis } \\
\text { (n: 16) }\end{array}$ & $\begin{array}{c}\text { Moderate- } \\
\text { severe } \\
\text { steatosis } \\
\text { (n: 11) }\end{array}$ & P value \\
\hline $\begin{array}{c}\text { LAL activity } \\
\text { nmol/spot/h } \\
\text { range }\end{array}$ & 1,14 & 0,89 & 0,86 & 0.1 \\
\hline $\begin{array}{c}\text { TRIGLYCERIDES } \\
\text { mg/dl } \\
\text { range }\end{array}$ & $0,7-2,8$ & $0,22-1,9$ & $0,34-1,2$ & 0.6 \\
\hline
\end{tabular}

Note: all values are expressed as mean values. ${ }^{*} \mathrm{P}<0,05$

1: Lysosomal Acid Lipase Deficiency.

"Finally, comparison between low LAL-A and normal LAL-A in patients with chronic liver disease showed that:

a) low LAL-A subjects had significantly higher triglycerides and uric acid levels than normal one (Table 2);

Table 2: $\mathrm{BMI}^{1}$ and laboratory features of low LAL-A ${ }^{2}$ (N 11) and normal LAL-A (N 40) subjects.

\begin{tabular}{|c|c|c|c|}
\hline Parameter & $\begin{array}{l}\text { Low lal-a } \\
\text { subjects }\end{array}$ & $\begin{array}{l}\text { Normal lal-a } \\
\text { subjects }\end{array}$ & $P$ value \\
\hline $\begin{array}{c}\mathrm{BMI} \mathrm{kg} / \mathrm{m}^{2} \\
\text { range }\end{array}$ & $\begin{array}{c}23,7 \\
15,6-29,6\end{array}$ & $\begin{array}{c}21,6 \\
16,5-35,8\end{array}$ & 0.26 \\
\hline $\begin{array}{l}\text { ALT UI/l } \\
\text { range }\end{array}$ & $\begin{array}{c}45.3+11.1 \\
5-109\end{array}$ & $\begin{array}{c}57.2+10.6 \\
11-318\end{array}$ & 0.56 \\
\hline $\begin{array}{l}\text { GGT UI/l } \\
\text { range }\end{array}$ & $\begin{array}{c}38.7+9.34 \\
\text { Nov-90 }\end{array}$ & $\begin{array}{c}29.4+6.64 \\
5-183\end{array}$ & 0.49 \\
\hline $\begin{array}{c}\text { TRIGLYCERIDES } \\
\mathrm{mg} / \mathrm{dl} \\
\text { range }\end{array}$ & $\begin{array}{c}129+30.2 \\
35-327\end{array}$ & $\begin{array}{l}86,4+7 \\
29-210\end{array}$ & $0.04^{*}$ \\
\hline $\begin{array}{l}\mathrm{T}-\mathrm{C}^{3} \mathrm{mg} / \mathrm{dl} \\
\text { range }\end{array}$ & $\begin{array}{c}161,5+39.5 \\
105-228\end{array}$ & $\begin{array}{c}145,4+33.4 \\
102-230\end{array}$ & 0.19 \\
\hline $\begin{array}{l}\mathrm{LDL}-\mathrm{C}^{4} \mathrm{mg} / \mathrm{dl} \\
\text { range }\end{array}$ & $\begin{array}{c}80,4+10.9 \\
40-148\end{array}$ & $\begin{array}{l}76+4.5 \\
31-146\end{array}$ & 0.68 \\
\hline $\begin{array}{l}\text { HDL-C }{ }^{5} \mathrm{mg} / \mathrm{dl} \\
\text { range }\end{array}$ & $\begin{array}{c}48+6.4 \\
26-86 \\
\end{array}$ & $\begin{array}{c}52+2.9 \\
31-96 \\
\end{array}$ & 0.47 \\
\hline $\begin{array}{l}\text { INSULIN } \mathrm{mU} / \mathrm{ml} \\
\text { range }\end{array}$ & $\begin{array}{c}12,7+10 \\
\text { Feb-31 }\end{array}$ & $\begin{array}{c}14,0+13 \\
\text { Feb-53 }\end{array}$ & 0.79 \\
\hline $\begin{array}{c}\text { HOMA INDEX } \\
\text { range }\end{array}$ & $\begin{array}{c}2,8+2.3 \\
0,56-6,38 \\
\end{array}$ & $\begin{array}{l}2,8+2.5 \\
0,4-7,14\end{array}$ & 0.96 \\
\hline $\begin{array}{l}\text { URIC ACID } \\
\mathrm{mg} / \mathrm{dl} \\
\text { range }\end{array}$ & $\begin{array}{c}5.4+0.4 \\
3.6-7.9\end{array}$ & $\begin{array}{c}3.7+0.2 \\
1.8-6.5\end{array}$ & $0.0002^{*}$ \\
\hline
\end{tabular}

Note: all values are expressed as mean values. ${ }^{*} \mathrm{P}<0,05$

1: Body Mass Index.

2: Lysosomal Acid Lipase Activity.

3: Total Cholesterol
4: LDL Cholesterol

5: HDL Cholesterol

b) WD patients with low LAL-A had BMI, T-C, triglycerides and uric acid levels higher then normal LAL-A WD patients (Table 3).

Table 3: BMI 1 and laboratory features of low LAL-A2 (N 6) and normal LAL-A (N 14) subjects within the group of patients with Wilson disease.

\begin{tabular}{|c|c|c|c|}
\hline Parameter & $\begin{array}{l}\text { Wd low lal-a } \\
\text { subjects }\end{array}$ & $\begin{array}{l}\text { Wd normal } \\
\text { lal-a subjects }\end{array}$ & $p$ value \\
\hline $\begin{array}{c}\mathrm{BMI} \mathrm{kg} / \mathrm{m}^{2} \\
\text { range }\end{array}$ & $\begin{array}{l}26,4+3.7 \\
22,6-31,3\end{array}$ & $\begin{array}{l}21,9+7 \\
18-34,1\end{array}$ & $0,04^{*}$ \\
\hline $\begin{array}{l}\text { ALT UI/l } \\
\text { range }\end{array}$ & $\begin{array}{c}61,8+36 \\
25-109\end{array}$ & $\begin{array}{c}64,1+60 \\
16-242\end{array}$ & 0,9 \\
\hline $\begin{array}{l}\text { GGT UI/l } \\
\text { range }\end{array}$ & $\begin{array}{c}43.2+13 \\
14-77 \\
\end{array}$ & $\begin{array}{c}30.3+12 \\
6-178 \\
\end{array}$ & 0.5 \\
\hline $\begin{array}{c}\text { TRIGLYCERIDES } \\
\mathrm{mg} / \mathrm{dl} \\
\text { range }\end{array}$ & $\begin{array}{c}193.2+38.7 \\
86-327\end{array}$ & $\begin{array}{c}113,7+10.4 \\
53-210\end{array}$ & $0,01^{*}$ \\
\hline $\begin{array}{l}\mathrm{T}-\mathrm{C}^{3} \mathrm{mg} / \mathrm{dl} \\
\text { range }\end{array}$ & $\begin{array}{c}181.3+15 \\
131-217\end{array}$ & $\begin{array}{l}139.4+9 \\
102-224\end{array}$ & $0,02^{*}$ \\
\hline 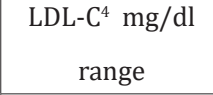 & $\begin{array}{c}104,8+12.7 \\
70-148\end{array}$ & $\begin{array}{c}75.1+8.4 \\
50-150\end{array}$ & 0.08 \\
\hline $\begin{array}{l}\mathrm{HDL}-\mathrm{C}^{5} \mathrm{mg} / \mathrm{dl} \\
\text { range }\end{array}$ & $\begin{array}{c}35.8+5.2 \\
24-53\end{array}$ & $\begin{array}{c}46.6+4.7 \\
33-78\end{array}$ & 0.21 \\
\hline $\begin{array}{l}\text { INSULIN } \mathrm{mU} / \mathrm{ml} \\
\text { range }\end{array}$ & $\begin{array}{l}14,52+5 \\
3,4-30,9\end{array}$ & $\begin{array}{l}18,39+5 \\
4,3-32,7 \\
\end{array}$ & 0,6 \\
\hline $\begin{array}{c}\text { HOMA INDEX } \\
\text { range }\end{array}$ & $\begin{array}{c}3,5+0.8 \\
0,56-6,38\end{array}$ & $\begin{array}{l}2,9+1.07 \\
0,91-8,83\end{array}$ & 0,7 \\
\hline $\begin{array}{l}\text { URIC ACID } \\
\mathrm{mg} / \mathrm{dl}\end{array}$ & $5.6+0.6$ & $3.8+0.4$ & $0,01^{*}$ \\
\hline
\end{tabular}

Note: all values are expressed as mean values. ${ }^{*} \mathrm{P}<0,05$

1: Body Mass Index.

2: Lysosomal Acid Lipase Deficiency.

3: Total Cholesterol.

4: LDL Cholesterol.

5: HDL Cholesterol.

\section{Discussion}

LAL-D is a rare autosomal recessive disease that should be researched in each child with a liver disease of unknown origin. According to current protocols, it should always be suspected in non-obese patients presenting with NAFLD and/or cryptogenic cirrhosis, unexplained persistently elevated liver transaminases or with elevation in LDL-C and decreased HDL-C. Guidelines suggest testing LAL activity using the DBS test [7]. Recently it was supposed that a secondary deficit of LAL may be linked to NAFLD in both adults and children. In general, it is known that a deficient enzymatic activity has a high impact on lipid metabolism, determining a significant increase of cardiovascular risk due to 
dyslipidemia and premature atherosclerosis, while cholesteryl esters and triglycerides stored in hepatocytes have an important role in inducing hepatic fibrosis [12].

Our study shows that low LAL activity has a role in liver disease other than LAL-D and in these patients it is inversely related to serum triglycerides and uric acid serum levels, two markers of metabolic syndrome. It must be underlined that in our cohort also the presence of steatosis at ultrasound is significantly related to low LAL activity, confirming a possible link of this enzyme activity with fatty liver and metabolic syndrome. Recently it has been demonstrated that dietary fructose intake increases serum uric acid concentrations and impairs lysosome-autophagy pathway resulting in increased hepatic steatosis in animal models [13]. In this study we prove for the first time an inverse correlation between LAL activity and uric acid serum levels that needs further evaluation in order to clarify the relationship between fructose assumption, LAL activity and NAFLD.

LAL activity seems not to be related with levels of ALT as suggested recently in adult NAFLD patients [8]. We could speculate that patients with liver disease and concomitant deficit of LAL may have a higher risk of metabolic syndrome and therefore a worst prognosis and disease progression. Furthermore the direct correlation between LAL activity and age of patients could suggest that liver disease progression over time may worst enzyme deficiency that in turn enhances lipid deposition in the liver. However it is difficult to establish if low LAL-A is a cause or an epiphenomenon of fatty liver and metabolic syndrome.

Another interesting finding of our study is the high prevalence of low LAL activity in patients with Wilson disease. In these patients LAL activity was related with higher values of BMI, cholesterol, triglycerides and uric acid serum levels. These findings, in association with hepatic steatosis which is frequently described in WD [14], may suggest an important role of LAL deficit in WD liver disease progression. In fact, in WD both serum lipoprotein alterations and fatty liver are common and share many pathophysiological mechanisms with LAL-D [8].

The earliest characteristic alterations of the liver pathology in WD include steatosis, which is sometimes indistinguishable from fatty liver due to metabolic syndrome. Recently it has been supposed that a genetic polymorphism in rs738409, in the patatinlike phospholipase domain (PNPLA3), may have a role in steatosis in WD patients. We could suppose that, as PNPLA3 polymorphism, also LAL deficiency may be a contributing factor that influences progression of WD in a subgroup of patients. This may explain, at least in part, the wide variability of phenotype and long-term course of liver disease among WD patients. Therefore LAL activity reduction could be a contributing factor in progression of fatty liver disease to cirrhosis not only in obese patients, as previously supposed $[15,16]$, but also in other categories of fatty liver, like WD. Clearly it is difficult to hypothesize if the improvement in LAL activity may translate into a reduction of fatty liver content. Therefore we are far enough to speculate about potential new targets for the enzyme-replacement.
On the basis of our findings we suggest to not limit DBS screening to non-obese children, but to include all obese children with NAFLD with a rapid and worst progression nonresponder to conventional lifestyle interventions. This hypothesis is also supported by two cases of obese children diagnosed with LAL-D [15].

We suggest reading our results from the point of view of lysosomal-autophagy pathway, which is a key process in hepatic lipid metabolism and steatosis [10]. Its dysregulation determines progression of fatty liver disease in patients with NAFLD and WD [16-18]. In particular, lipid droplets (LD) are delivered to lysosomes via autophagy, where LAL acts to hydrolyze LD cholesteryl esters to generate free cholesterol [17]. Therefore; it could be interesting to analyze the potential relationship between liver disease progression, autophagy dysregulation and LAL activity deficiency in a larger series of patients with liver disease. The main limitation of our study is that LAL activity evaluation by DBS is exposed to problems related to storage and shipment, that may greatly influence final results. For this reason we introduced a second evaluation to confirm positive finding by DBS. Moreover it is desirable to implement this analysis to reach some definitive conclusions, also considering in vitro/in vivo model of fatty liver in which to analyze LAL activity and its impact on disease progression.

\section{Conclusion}

Various liver diseases are associated with a secondary LAL deficiency that is inversely related to serum triglycerides and uric acid serum levels, two markers of metabolic syndrome, and correlates also with grade of steatosis. A high prevalence of LAL-d is observed in patients with Wilson Disease, especially in those with dyslipidaemia and steatosis. These results could be the first step to consider LAL activity as a biomarker of liver disease progression, with potentially implications in prognostic but also therapeutic fields.

\section{References}

1. Bernstein DL, Hulkova H, Bialer MG, Desnick RJ (2013) Cholesteryl ester storage disease: review of the findings in 135 reported patients with an underdiagnosed disease. J Hepatol 58(6): 1230-1243.

2. Reiner Z, Guardamagna O, Nair D, Soran H, Hovingh K, et al. (2014) Lysosomal acid lipase deficiency -An under-recognised cause of dyslipidaemia and liver dysfunction. Atherosclerosis 235(1): 21-30.

3. Hulkova H, Elleder M (2012) Distinctive hisyopathological features that support a diagnosis of cholesterol ester storage disease in liver biopsy specimens. Histopathology 60(7): 1107-1113.

4. Zhang B, Porto AF (2013) Cholesteryl ester storage disease: protean presentations of lysosomal acid lipase deficiency. J Pediatr Gastroenterol Nutr 56(6): 682-685.

5. Boldrini R, Devito R, Biselli R, Filocamo M, Bosman C (2004) Wolman disease and cholesteryl ester storage disease diagnosed by histological and ultrastructural examination of intestinal and liver biopsy. Pathol Res Pract 200(3): 231-240.

6. Elleder M, Chlumska A, Hyanek J, Poupĕtová H, Ledvinová J, et al. (2000) Subclinical course of cholesteryl ester storage disease in an adult with hypercholesterolemia, accelerated atherosclerosis, and liver cancer. J Hepatol 32(3): 528-534.

7. Hamilton J, Jones I, Srivastava R, Galloway P (2012) A new method for the measurement of lysosomal acid lipase in dried blood spots using the inhibitor Lalistat 2. Clin Chim Acta 413(15-16): 1207-1210. 
8. Baratta F, Pastori D, Polimeni L, Tozzi G, Violi F, et al. (2015) Does lysosomal acid lipase reduction play a role in adult Non-Alcoholic Fatty Liver Disease? Int J Mol Sci 16(12): 28014-28021.

9. Selvakumar PK, Kabbany MN, Lopez R, Tozzi G, Alisi A, et al. (2016) Reduced lysosomal acid lipase activity-A potential role in the pathogenesis of non alcoholic fatty liver disease in pediatric patients. Digestive and Liver Disease 48 (8): 909-913.

10. Shteyer E, Villenchik R, Mahamid M, Nator N, Safadi R, et al. (2016) Low serum lysosomal acid lipase activity correlates with advanced liver disease. Int J Mol Sci 17(3): 312.

11. Kuczmarski RJ, Ogden CL, Guo SS, Flegal KM, Guo SS, et al. (2000) CDC growth charts: United States. Adv Data 314: 1-27.

12. Burton BK, Deegan PB, Enns GM (2015) Clinical Features of Lysosomal Acid Lipase Deficiency-a Longitudinal Assessment of 48 Children and Adults. J Pediatr Gastroenterol Nutr 61(6): 619-625.

13. Mosca A, Nobili V, De Vito R (2017) Serum acid concentrations and fructose consumption are independently associated with NASH in children and adolescents. J Hepatol 66(5): 1031-1036.

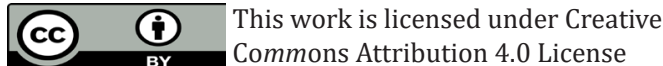

Submission Link: https://biomedres.us/submit-manuscript.php
14. Liggi M, Murgia D, Civolani A (2013) The relationship between copper and steatosis in Wilson's disease. Clin Res Hepatol Gastroenterol 37(1): 36-40.

15. Himes RW, Barlow SE, Bove K (2016) Lysosomal acid lipase deficiency unmasked in two children with nonalcoholic fatty liver disease. Pediatrics 138(4).

16. Skop V, Cahovà M, Papackzova Z (2012) Autophagy-lysosomal pathway is involved in lipid degradation in rat liver. Phisiol Res 61(3): 287-297.

17. Ouimet M, Franklin V, Mak E (2011) Autophagy regulates cholesterol efflux from macrophage foam cells via lysosomal acid lipase. Cell Metab 13(6): 655-667.

18. Polishchuk EV, Concilli M, Iacobacci S (2014) Wilson disease protein ATP7B utilizes lysosomal exocytosis to maintain copper homeostasis. Dev Cell 29(6): 686-700.

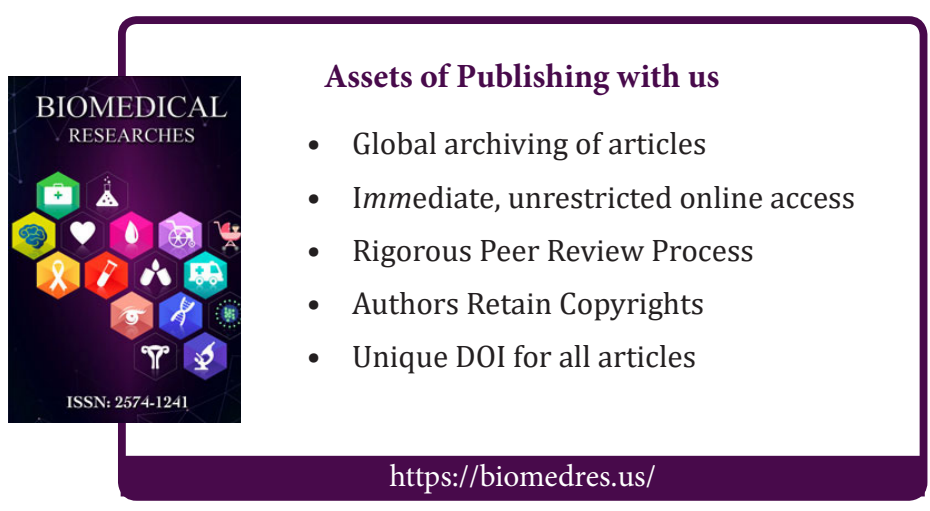

